\title{
Acknowledgment to Reviewers of Journal of Cardiovascular Development and Disease in 2020
}

\section{JCDD Editorial Office}

Citation: JCDD Editorial Office.

Acknowledgment to Reviewers of

Journal of Cardiovascular Development and Disease in 2020. J. Cardiovasc. Dev. Dis. 2021, 8, 7. https://doi.org/ $10.3390 /$ jcdd 8020007

Published: 22 January 2021

Publisher's Note: MDPI stays neutral with regard to jurisdictional claims in published maps and institutional affiliations.

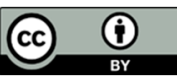

Copyright: (๑) 2021 by the author. Licensee MDPI, Basel, Switzerland. This article is an open access article distributed under the terms and conditions of the Creative Commons Attribution (CC BY) license (http://creativecommons.org/licenses/by/4.0/).

MDPI AG, St. Alban-Anlage 66, 4052 Basel, Switzerland

Peer review is the driving force of journal development, and reviewers are gatekeepers who ensure that Journal of Cardiovascular Development and Disease maintains its standards for the high quality of its published papers. Thanks to the cooperation of our reviewers, in 2020, the median time to first decision was 20.5 days and the median time to publication was 42 days. The editors would like to express their sincere gratitude to the following reviewers for their precious time and dedication, regardless of whether the papers were finally published:

Adler, Eric

Ahmad, Shaad

Aiello, Vera

Amack, Jeffrey

Amri, Ibtihal Al

Anderson, Robert

Angelini, Paolo

Astiazaran-Garcia, Humberto

Azhar, Mohamad

Bakkers, Jeroen P.W.

Baldini, Antonio

Ballmann, Christopher

Bamforth, Simon D.

Barrett, Hugh

Belo, José António

Beyer, Eric C.

Bittel, Douglas C.

Bogomolovas, Julijus

Borghini, Andrea

Brčić Karačonji, Irena

Bronner, Marianne

Buch, Eric

Buchholz, John

Cabezas, Jose Montero

Calissendorff, Jan

Calvo, David

Campione, Marina

Campuzano, Oscar

Capoccia, Massimo

Caroleo, Maria Cristina

Casini, Alessandro

Ceolotto, Giulio
Chaudhry, Bill

Chen, Chao

Chen, Jau-nian

Chevret, Edith

Ciccone, Marco

Collins, Michelle M.

Colussi, Gian Luca

Conway, Simon

Cornell, Robert

Crocini, Claudia

Eller, Kathrin

Ezhov, Marat

Faletra, Francesco

Favaloro, Emmanuel J.

Fukunaga, Naoto

Galatou, Eleftheria

Garcia-Jares, Carmen

Gatzoulis, Konstantinos A.

Gentile, Marco

Giordano, Raffaele

Giovannoni, Roberto

Gomez-Sanchez, Elise

Gorini, Francesca

Gragnano, Felice

Grau, Marijke

Groot, A.C. Gittenberger-de

Hafiane, Anouar

Harenberg, Job F.

Henderson, Deborah

Hiebert, Linda

Holaj, Robert

Holzmann, Klaus 
Horowitz, John D.

Houyel, Lucile

Hsu, Edward

Hwang, Chueh-Lung

Ichibori, Yasuhiro

Imamura, Teruhiko

Irwin, David C.

Ishibashi, Nobuyuki

Jänig, Wilfrid

Jaźwińska, Anna

Jean Francois, Schved

Jensen, Bjarke

Jiao, Kai

Jinnouchi, Hiroyuki

John, Pepper

Julius, Ulrich

Kasai, Takatoshi

Keller, Bradley

Kelly, Robert

Kocica, Mladen J.

Kostner, Gert M.

Kowalski, William J.

Kruithof, Boudewijn

Kurath-Koller, Stefan

Kutikhin, Anton G.

Kuwata, Shingo

Kwon, Yu-jin

Latif, Najma

Lee, Wei-Chieh

Leire, Unzué

Levi, Giovanni

Lin, Wen

$\mathrm{Lu}$, Quanlong

Lynge, Thomas Hadberg

Ma, Hong

Machon, Ondrej

Männer, Jörg

Mariotti, Alessandro

Markovic, Sinisa

Marrs, James A.

Marsan, Nina Ajmone

Masarone, Daniele

Masella, Roberta

Maslen, Cheryl L.

Mauro, Feola

Mavrogeni, Sophie

Messonnier, Laurent A.

Mestroni, Luisa

Metzinger, Laurent

Meuris, Bart

Miyachi, Hideki
Miyagawa-Tomita, Sachiko

Mohsin, Sadia

Mongillo, Marco

Morrow, Bernice E.

Mozos, Ioana

Norris, Russell

Panayiotou, Andrie G.

Pekkan, Kerem

Pepi, Mauro

Poelmann, Robert

Prickett, Tim

Przybylowicz, Katarzyna

Puślecki, Mateusz

Rauch, Bernhard

Rugonyi, Sandra

Ruiz, Santiago

Sampietro, Tiziana

Sarecka-Hujar, Beata

Sato, Akira

Scambler, Peter

Schlüter, Klaus-Dieter

Schoenhagen, Paul

Senesi, Pamela

Shiraishi, Isao

Shiratori, Hidetaka

Stapleton, Phoebe

Stefani, Laura

Stoffman, Jayson

Suñer, Damian Heine

Surdacki, Andrzej

Tan, Bee Kang

Thomas, Shane

Toma, Milan

Tranter, Michael

Tretter, Justin T.

Tsang, Michael

Tseng, Elaine E.

Turiel, Maurizio

Van Den Hoff, Maurice

Van Heesch, Sebastiaan

Vancheri, Federico

Vaškelytė, Jolanta

Vecoli, Cecilia

Velasco, Carlos E.

Wang, Jiongwei

Wang, $\mathrm{Xu}$

Watanabe, Eiichi

Wessells, Robert

Wigle, Jeffrey

Wilczek, Piotr

Wingert, Rebecca 
Xin, Mei

$\mathrm{Xu}$, Xiaolei

Yang, Zhongzhou

Yutzey, Katherine
Žáček, Pavel

Zaffran, Stephane

Zhang, Jianyi 